\title{
Comprehensive Laboratory Evaluation of a Lateral Flow AsSAY FOR THE Detection OF YeRSINIA PESTIS
}

Kristin W. Prentice, Lindsay DePalma, Jason G. Ramage, Jawad Sarwar, Nishanth Parameswaran, Jeannine Petersen, Brook Yockey, John Young, Mrinmayi Joshi, Nagarajan Thirunavvukarasu, Ajay Singh, Carol Chapman, Julie R. Avila, Christine A. Pillai, Gowri Manickam, Shashi K. Sharma, Stephen A. Morse, Kodumudi Venkat Venkateswaran, Kevin Anderson, David R. Hodge, and Segaran P. Pillai

We conducted a comprehensive, multiphase laboratory evaluation of the Plague BioThreat Alert ${ }^{\circledR}$ (BTA) test, a lateral flow immunoassay (LFA), for the rapid detection of Yersinia pestis. The study was conducted in 7 phases at 2 sites to assess the performance of the LFA. The limit of detection (LOD) was determined using both a virulent and avirulent strain of $Y$. pestis, CO99-3015 (10 $\mathrm{CFU} / \mathrm{ml})$ and A1 $122\left(10^{4} \mathrm{CFU} / \mathrm{ml}\right)$, respectively. In the other phases, $18 Y$. pestis strains, 20 phylogenetic near-neighbor strains, 61 environmental background microorganisms, 26 white powders, and a pooled aerosol sample were also tested. A total of 1,110 LFA test results were obtained, and their analysis indicates that this LFA had a sensitivity of $97.65 \%$ and specificity of $96.57 \%$. These performance data are important for accurate interpretation of qualitative results arising from testing suspicious white powders and aerosol samples in the field. Any positive specimen in this assay is considered presumptive positive and should be referred to the Centers for Disease Control and Prevention Laboratory Response Network for additional testing, confirmation, and characterization for an appropriate public health response.

Keywords: Yersinia pestis, Plague, Lateral flow immunoassay, Rapid detection

Kristin W. Prentice, MS, is an Associate, Booz Allen Hamilton, Rockville, MD. Lindsay DePalma, MS, is a Staff Life Scientist, Booz Allen Hamilton, McLean, VA. Jason G. Ramage, MS, MBA, PMP, is Assistant Vice Chancellor for Research and Innovation and Director of Research Compliance, University of Arkansas, Fayetteville, AR. Jawad Sarwar, MS, is a Senior Research Scientist, and Nishanth Parameswaran and Mrinmayi Joshi, MS, are Research Scientists; all at Omni Array Biotechnology, Rockville, MD. Jeannine Petersen, PhD, Brook Yockey, and John Young are Microbiologists; all with DHHS/CDC/OID/NCEZID/DVBD/BDB, Fort Collins, CO. Nagarajan Thirunavvukarasu, PhD, is an ORISE Fellow; Christine A. Pillai and Gowri Manickam, PhD, are ORISE Fellow Research Scientists; and Shashi K. Sharma, PhD, is a Research Microbiologist; all at the FDA Center for Food Safety and Applied Nutrition, Molecular Methods Development Branch, Division of Microbiology, Office of Regulatory Science, College Park, MD. Ajay Singh, PhD, is a Research Scientist, Laulima Government Solutions, Contractor Support to USAMRICD, Neurobiological Toxicology Branch, Analytical Toxicology Division, Aberdeen Proving Ground, MD. Carol Chapman, MS, is a Microbiologist, Geneva Foundation, Contractor Support to the Naval Medical Research Center, Silver Spring, MD. Julie R. Avila, MS, is a Scientific Associate, Lawrence Livermore National Laboratory, Biosciences and Biotechnology Division, Livermore, CA. Stephen A. Morse, $\mathrm{MSPH}, \mathrm{PhD}$, is a Senior Advisor, CDC Division of Select Agents and Toxins, and is currently with IHRC, Inc., Atlanta, GA. Kodumudi Venkat Venkateswaran, PhD, is Chief Scientist, Tetracore, Inc., Rockville, MD. Kevin Anderson, PhD, and David R. Hodge, PhD, are Program Managers, Science and Technology Directorate, US Department of Homeland Security, Washington, DC. Segaran P. Pillai, PhD, is Director, Office of Laboratory Science and Safety, FDA Office of the Commissioner, Silver Spring, MD.

(C) Kristin W. Prentice, et al., 2019; Published by Mary Ann Liebert, Inc. This Open Access article is distributed under the terms of the Creative Commons License (http://creativecommons.org/licenses/by/4.0), which permits unrestricted use, distribution, and reproduction in any medium, provided the original work is properly credited. 
$Y$ ERSINIA PESTIS is the causative agent of plague. It is a Gram-negative, nonmotile, non-spore forming coccobacillus, which is urease and indole negative. ${ }^{1-7}$ As a facultative anaerobe belonging to the Yersiniaceae, $Y$. pestis evolved from Yersinia pseudotuberculosis between 2,600 and 28,000 years ago, after the acquisition of $2 Y$. pestis-specific plasmids: pMT1 and pPCP1. ${ }^{8,9} Y$. pestis grows at $28^{\circ} \mathrm{C}$, which is the normal body temperature for fleas, and at $37^{\circ} \mathrm{C}$, the normal body temperature for humans. ${ }^{1,4,6,7}$ In the laboratory, the bacterium can grow on standard microbiologic media. ${ }^{4}$ Observable growth is present at 24 to 48 hours, although colonies are smaller than those seen with other bacteria. ${ }^{4,7}$ In the wild, $Y$. pestis infects rodents, including rats, squirrels, and prairie dogs. ${ }^{1,4,10,11}$ Infection usually results from being bitten with a $Y$. pestis-infected vector such as the Oriental rat flea (Xenopsylla cheopis). ${ }^{1,3,4,10,12-15}$ Less commonly, infection can occur through handling infected animals or from inhaling infective respiratory droplets or aerosols. ${ }^{3}$

All 3 pathogenic species of Yersinia ( $Y$. pestis, Y. pseudotuberculosis, and Yersinia enterocolitica) carry the $70 \mathrm{kbp}$ pCD1 plasmid, which encodes for a type III secretion system and Yersinia outer membrane proteins (Yops) that enable these bacteria to evade the host immune system. The pCD1 plasmid is not found in nonpathogenic Yersinia species. ${ }^{9,13,16-18}$ Virulent strains of $Y$. pestis also possess 2 additional plasmids: the $90 \mathrm{kbp}$ pMT1 plasmid, alternatively known as pFra, which encodes the F1 capsular protein, and murine toxin. ${ }^{19}$ The $\mathrm{F} 1$ capsule enables $Y$. pestis to resist engulfment by neutrophils and macrophages. ${ }^{1,12}$ The F1 gene is temperature regulated and expressed at $\geq 33^{\circ} \mathrm{C}$. The $9.5 \mathrm{kbp}$ pPCP1 plasmid, also known as pPla or pPst, encodes for plasminogen-activating factor as well as pestin and coagulase. ${ }^{10,19}$ In the laboratory, the plasmids can be lost during storage or on subculture. ${ }^{19,20}$

There are 3 clinical forms of plague: bubonic, septicemic, and pneumonic. The most common form is bubonic plague, acquired through the bite of an infected flea, which accounts for approximately $80 \%$ to $95 \%$ of all cases worldwide. Infected humans develop regional lymph node swelling and fever; bubonic plague is not transmissible from person to person and has a mortality rate of approximately $50 \%$ if untreated. Following entry into the body, $Y$. pestis is phagocytized by both neutrophils and macrophages; however, the bacteria survive and replicate in macrophages. Following infiltration of the lymph nodes, bacteria may enter the bloodstream, resulting in septicemic plague, which can also be caused directly via wound exposure. ${ }^{4-6,14,15,21-23}$

Pneumonic plague is far more dangerous but is very rare. Secondary pneumonic plague may result from the dissemination of bacteria to the lungs in cases of bubonic or septicemic plague, leading to severe bronchopneumonia, chest pain, dyspnea, cough, and hemoptysis. Primary pneumonic plague results from the direct inhalation of airborne droplets or aerosols of $Y$. pestis and is the clinical form most likely to occur following an aerosol release in a bioterrorism attack. Pneumonic plague is transmissible from person to person via airborne droplets. Infected individuals experience a 2- to 4day incubation period, followed by rapid onset of chills, fever, general malaise, increased heart and respiratory rates, elevated body temperature, and a cough that becomes bloody as the disease progresses. During the terminal stage of the disease, patients experience hemorrhagic necrosis, acute respiratory failure, sepsis, and circulatory collapse. Diagnosis is based on immunostaining, PCR, and culture. In the absence of prompt treatment with antibiotics, pneumonic plague has a mortality rate that approaches $100 \%$. Recommended treatment involves a 10- to 14-day regimen with an antibiotic such as streptomycin, gentamicin, ciprofloxacin, levofloxacin, or doxycycline.

First responders often encounter unknown and suspicious white powders in the field, and it is important to quickly evaluate them for the possible presence and identification of biological threat agents. The results of these evaluations can inform decisions to initiate public safety actions, including area evacuation, facility closure to prevent additional exposures, decontamination and initiation of medical countermeasures for potentially exposed individuals, collection of samples for law enforcement and public health purposes, sample transfer to a reference laboratory for immediate testing, and containment of contamination to prevent secondary dissemination or reaerosolization. In order to support first responders with the appropriate tools to carry out their mission, there is a need to understand performance of rapid assays for screening suspicious white powders.

The purpose of the present study was to evaluate the sensitivity, specificity, limit of detection (LOD), reproducibility, and limitations of the Plague BioThreat Alert test (Tetracore, Inc., Rockville, MD) as pertains to its use in the field to screen for the presence of $Y$. pestis. The goal of this study was to provide an understanding of assay performance, including the likelihood of false-negative results (assay is negative, but the target analyte is present at a concentration at or above the LOD), false-positive results (assay is positive, but the target analyte is not present in the sample), the limit of detection, and reproducibility, so that appropriate and effective decisions can be made by first responders to support public safety actions while avoiding unnecessary fear, panic, and costly civil disruptions.

This study was designed and executed through an interagency collaboration with participation from subject matter experts from the Department of Homeland Security (DHS), the Department of Health and Human Services (HHS), the Department of Justice (DOJ), the US Department of Agriculture (USDA), and the US Secret Service (USSS).

\section{Materials and Methods}

\section{Biosafety Considerations}

Strains used in this study were handled with appropriate biosafety conditions in accordance with the 5 th edition of 
Biosafety in Microbiological and Biomedical Laboratories $(\mathrm{BMBL})^{24}$ and the Federal Select Agent Regulations.

\section{Plague BTA Test and BioThreat Alert Reader $M X$}

The Plague BTA test, which employs antibodies to detect the presence of the F1 capsular antigen, BioThreat Alert Reader MX (BTA Reader), and BTA Sample Buffer (BTA buffer) were obtained from Tetracore, Inc. (Rockville, MD). The performance of the Plague LFA and BTA Reader was evaluated at 2 test sites: (1) the Centers for Disease Control and Prevention (CDC), Fort Collins, CO, and (2) Omni Array Biotechnology Corporation, Rockville, MD. Samples for phases 1, 2, and 7 were prepared and tested at CDC, and samples for phases 3 to 6 were prepared and stored at $4^{\circ} \mathrm{C}$ at Omni Array until use, then analyzed by personnel from DHS Science and Technology (S\&T) and FDA Center for Food Safety and Applied Nutrition (CFSAN), according to a standard protocol provided by the manufacturer.

Plague BTA LFA results were read both visually and with a BTA Reader, according to the directions provided by the manufacturer-that is, between 15 and 30 minutes after adding the sample $(0.150 \mathrm{~mL})$ to the BTA LFA. Samples with readings of $<200$ were considered negative, and test strips that failed to develop a control line were noted and discarded. Positive or negative determinations were based on the reader result. BTA buffer was used as a negative control. $Y$. pestis strain A1122 (avirulent, select agent exempt strain) was used as a positive control at a concentration 2 logs above LOD $\left(10^{7}\right.$ to $\left.10^{8}\right)$ at both test sites.

\section{Culture Preparation}

All bacterial isolates used in phases 1 and 2 were grown and prepared at CDC. Isolates were inoculated onto $6 \%$ sheep blood agar (SBA) and incubated at $35^{\circ} \mathrm{C}$ for 24 hours. Isolates were subcultured to confirm purity and then used to prepare cell suspensions. Suspensions were prepared in $2 \mathrm{~mL}$ of $0.85 \%$ sterile saline and lightly vortexed. Stock suspensions were adjusted to an OD600 absorbance of 1.0 (5.5 x $10^{9} \mathrm{CFU} / \mathrm{mL}$ ) using a Microscan turbidity meter (Dade Behring, Inc., Deerfield, IL). The CFU/mL for a $Y$. pestis cell suspension with an OD600 of 1.0 was determined by colony counts, and this absorbance was subsequently used for preparing for cell suspensions of assigned concentrations.

\section{Phase 1: Linear Dynamic Range and Repeatability Study}

The linear dynamic range of the Plague LFA was determined using both the virulent (CO99-3015) and avirulent (A1122) strains of $Y$. pestis. Bacterial suspensions for testing were prepared by diluting the stock suspension 10 - fold dilution in BTA buffer to achieve concentrations of $10^{8}-10^{9} \mathrm{CFU} / \mathrm{mL}, 10^{7}-10^{8} \mathrm{CFU} / \mathrm{mL}, 10^{6}-10^{7} \mathrm{CFU} / \mathrm{mL}$, $10^{5}-10^{6} \mathrm{CFU} / \mathrm{mL}, 10^{4}-10^{5} \mathrm{CFU} / \mathrm{mL}, 10^{3}-10^{4} \mathrm{CFU} / \mathrm{mL}$, and $10^{2}-10^{3} \mathrm{CFU} / \mathrm{mL}$. Each dilution was quantified by plating $0.1 \mathrm{~mL}$ dilutions in triplicate onto SBA agar plates and counting colonies after 48 hours' incubation at $35^{\circ} \mathrm{C}$. For testing, each suspension was lightly vortexed and immediately tested by adding $0.150 \mathrm{~mL}$ to the sample well of a test strip. Each concentration was tested 5 times, and the results read both visually and with 1 of 2 BTA Readers. The lowest concentration that yielded positive results in 5 out of 5 tests was further evaluated with an additional 120 test strips (repeatability study), with results read both visually and with 1 of 2 BTA Readers.

\section{Phase 2: Inclusivity Panel}

In order to determine whether the Plague LFA assay could detect diverse strains of $Y$. pestis, a total of $18 Y$. pestis strains (Table 1) were grown at $35^{\circ} \mathrm{C}$ for 24 hours. Bacterial suspensions were prepared and diluted in BTA buffer to a final concentration of $10^{7}$ to $10^{8} \mathrm{CFU} / \mathrm{mL}$ (2 logs above the LOD) and vortexed, and a $0.150-\mathrm{mL}$ volume was added to the sample port of 5 Plague BTA test strips. Results were read both visually and with 1 of 2 BTA Readers.

\section{Phase 3: Near Neighbor Panel}

Suspensions of 20 phylogenetic near neighbors of $Y$. pestis (Table 2) were prepared and diluted in BTA buffer to a concentration of $10^{8}$ to $10^{9} \mathrm{CFU} / \mathrm{mL}$ (3 logs above the LOD). After vortexing, a $0.150-\mathrm{mL}$ volume of each suspension was added to the sample wells of 5 test strips. Results were read both visually and with 2 BTA Readers.

\section{Phase 4: Environmental Background Panel}

The 61 diverse environmental background organisms that were selected based on the recommendations of a panel of subject matter experts are listed in Table $3 .^{25}$ Each organism was inoculated onto optimal solid medium and incubated under appropriate conditions for 24 to 48 hours. A single, isolated colony was selected and inoculated onto a second plate and incubated for 1 to 6 days, depending on the organism and its growth conditions. Plates were then sealed with parafilm, coded, and stored at $4^{\circ} \mathrm{C}$ until use. For testing, several colonies were removed and suspended in $4 \mathrm{~mL}$ BTA buffer to a final concentration of $10^{9}$ to $10^{10} \mathrm{CFU} / \mathrm{mL}$ ( 4 logs above LOD) and $0.150 \mathrm{~mL}$ added to the sample wells of 5 Plague LFAs.

\section{Phase 5a: White Powder Panel}

A stakeholder panel consisting of representatives from state public health laboratories, CDC, DOD, EPA, the FBI, and 
Table $1 . Y$. pestis inclusivity strains

\begin{tabular}{|c|l|l|}
\hline S. No. & \multicolumn{1}{|c|}{ Species } & \multicolumn{1}{c|}{ Strain ID } \\
\hline 1 & Yersinia pestis biovar antiqua & UG05-0454 \\
\hline 2 & Yersinia pestis biovar antiqua & Antiqua \\
\hline 3 & Yersinia pestis biovar antiqua & Nepal 516 \\
\hline 4 & Yersinia pestis biovar mediaevalis & Nicholisk 51 \\
\hline 5 & Yersinia pestis biovar mediaevalis & KIM 10 \\
\hline 6 & Yersinia pestis biovar mediaevalis & PyH1R3 \\
\hline 7 & Yersinia pestis biovar mediaevalis & PKH-10 \\
\hline 8 & Yersinia pestis biovar orientalis & CA00-2641 \\
\hline 9 & Yersinia pestis biovar orientalis & AZ94-0666 \\
\hline 10 & Yersinia pestis biovar orientalis & NM99-0030 \\
\hline 11 & Yersinia pestis biovar orientalis & ZE94-2122 \\
\hline 12 & Yersinia pestis biovar orientalis & P. Exu 2 \\
\hline 13 & Yersinia pestis biovar orientalis & A1122 \\
\hline 14 & Yersinia pestis biovar orientalis & EV 76 \\
\hline 15 & Yersinia pestis biovar orientalis & CO99-3015 OR CO92 \\
\hline 16 & Yersinia pestis biovar mediaevalis & Pestoides A \\
\hline 17 & Yersinia pestis biovar antiqua & Pestoides F \\
\hline 18 & Yersinia pestis biovar antiqua & \\
\hline
\end{tabular}

the commercial sector identified 26 white powders (Table 4) that were commonly encountered by first responders and CDC Laboratory Response Network (LRN) reference laboratories. $^{25,26}$ These powders were evaluated for their ability to affect the performance of the assay. Ten milligrams $(10 \mathrm{mg})$ of each powder was suspended in $1.00 \mathrm{~mL}$ of BTA buffer (final concentration $10 \mathrm{mg} / \mathrm{mL}$ ) and vortexed for 10 seconds. The suspension was allowed to settle for at least 5 minutes before $0.150 \mathrm{~mL}$ aliquots of the supernatant was removed and added to the sample wells of 5 Plague LFAs.

\section{Phase 5b: White Powder Spiked with Y. pestis strain A1122}

The 26 white powders tested in Phase 5a were spiked with $Y$. pestis strain A1122 and tested to determine if the white powders affected detection of $Y$. pestis by the LFA. Ten milligrams of each white powder were suspended in $0.900 \mathrm{~mL}$ of BTA buffer, and $0.100 \mathrm{~mL}$ of a suspension of $Y$. pestis strain A1122 was added to a final concentration of $10^{6}$ to $10^{7} \mathrm{CFU} /$ $\mathrm{mL}(1 \mathrm{log}$ above the LOD). Each tube was vortexed for 10 seconds. The suspensions were allowed to settle for at least 5 minutes; then $0.150 \mathrm{~mL}$ aliquots of the supernatant were removed and added to the sample wells of 5 LFAs to understand the degree, if any, to which the powder inhibited detection of $Y$. pestis.

\section{Phase 6a: Environmental Filter Extract}

A pooled aerosol filter extract (from 30 different filters), which contained $6 \mu \mathrm{g}$ protein $/ \mu \mathrm{L}$, was prepared as previously described $^{25}$ and shipped to the test site. Operators added $1.00 \mathrm{~mL}$ BTA buffer to $1.00 \mathrm{~mL}$ extract. After mixing for 10 seconds, the suspension was allowed to settle for at least 5 minutes, and $0.150 \mathrm{~mL}$ aliquots of supernatant $(450 \mu \mathrm{g}$ total protein) were added to the sample wells of 5 Plague LFAs.

\section{Phase 6b: Environmental Filter Extract Spiked with Y. pestis}

The pooled aerosol filter extract was spiked with $Y$. pestis strain A1122, at a final concentration of $10^{6}$ to $10^{7} \mathrm{CFU} / \mathrm{mL}$, and retested. After mixing for 10 seconds, the suspension was allowed to settle for at least 5 minutes, followed by removal of $0.150 \mathrm{~mL}$ aliquots of the supernatant for testing. The spiked pooled filter extract was tested a total of 5 times.

\section{Phase 7: Temperature Dependent Expression of F1 Capsular Antigen}

$Y$. pestis strains C099-3015 (Orientalis), Nepal 516 (Antiqua), and PyH1R3 (Mediaevalis), Pestoides A (Pestoides) were grown to test detection of temperature-dependent 
PRENTICE ET AL

Table 2. Y. pestis Near Neighbor Panel

\begin{tabular}{|c|c|c|c|c|}
\hline S. No. & Strain Name & Other Identifier & Biovar/ Serotype & Genome Homology \\
\hline 1 & Yersinia enterocolitica & ATCC 9610 & $0: 8$; Biovar 1 & $98 \%$ \\
\hline 2 & Yersinia enterocolitica & ATCC 700823 & 0:9: Biovar 2 & \\
\hline 3 & Yersinia enterocolitica & ATCC 700822 & $0: 3$; Biovar 4 & \\
\hline 4 & Yersinia enterocolitica subs. palearctica & DSM 13030 & & \\
\hline 5 & Yersinia entomophaga & DSM 22339 & & \\
\hline 6 & Yersinia massiliensis & DSM 21859 & & \\
\hline 7 & Yersinia nurmii & DSM 22296 & & \\
\hline 8 & Yersinia pekkanenii & DSM 22769 & & \\
\hline 9 & Yersinia pseudotuberculosis & ATCC 6902 & $1 \mathrm{a}$ & $99 \%$ \\
\hline 10 & Yersinia pseudotuberculosis & ATCC 13979 & 4 & $99 \%$ \\
\hline 11 & Yersinia pseudotuberculosis & ATCC 6905 & $2 b$ & $99 \%$ \\
\hline 12 & Yersinia intermedia & ATCC 33645 & & NA \\
\hline 13 & Yersinia frederiksenii & ATCC 29912 & & NA \\
\hline 14 & Yersinia aldovae & ATCC 35236 & Type strain & NA \\
\hline 15 & Yersinia aleksiciae & $\begin{array}{l}\text { Y159; CCUG 52872; DSM 14987; } \\
\text { LMG 22254; WA758 }\end{array}$ & & NA \\
\hline 16 & Yersinia bercovieri & $\begin{array}{l}\text { ATCC 43970; CDC 2475-87; CNY } \\
\text { 7506; WAIP 208; DSM 18528; CIP } \\
\text { 103323; CCUG } 26329\end{array}$ & & NA \\
\hline 17 & Yersinia kristensenii & ATCC 29911; CDC YE1474 & & NA \\
\hline 18 & Yersinia mollaretti & $\begin{array}{l}\text { ATCC 43969; CDC 2465-87; CNY } \\
\text { 7263; WAIP 204; CCUG 26331; CIP } \\
\text { 103324; DSM } 18520\end{array}$ & & NA \\
\hline 19 & Yersinia rohdei & $\begin{array}{l}\text { ATCC 43380; CDC 3022-85; CIP } \\
\text { 103163; DSM 18270; LMG 8454; } \\
\text { CCUG 38833; Aleksic H271/78 }\end{array}$ & & \\
\hline 20 & Yersinia ruckeri & $\begin{array}{l}\text { ATCC 29473; CDC 2396-61; NCIMB } \\
\text { 2194; CCM 6093; CCUG 14190; } \\
\text { CIP 82.80; DSM 18506; LMG } \\
\text { 21879; NCIMB 2194; NCTC } 12986\end{array}$ & & NA \\
\hline
\end{tabular}

expression of $Y$. pestis F1 capsular antigen. Strains were inoculated onto $6 \% \mathrm{SBA}$ and incubated either at $25^{\circ} \mathrm{C}$ or $35^{\circ} \mathrm{C}$ for 24 hours. Plates were incubated at $35^{\circ} \mathrm{C}$ for 24 hours followed by $25^{\circ} \mathrm{C}$ for an additional 4 days prior to testing. Cell suspensions were prepared in $2 \mathrm{~mL}$ of $0.85 \%$ sterile saline, lightly vortexed for homogenization, and adjusted to an absorbance of $1.0\left(5.5 \times 10^{9} \mathrm{CFU} / \mathrm{mL}\right)$ as previously described. Suspensions for testing were prepared by diluting $10-$ fold in BTA buffer and lightly vortexing immediately prior to testing. A concentration of 1 above the LOD was tested in 20 replicates at each temperature for each strain.

\section{Statistical Analysis}

Titration curves, Receiver Operator Characteristic Curves (ROC) based on BTA Reader values were made using
GraphPad Prism version 7.04 for Windows (GraphPad Software, La Jolla, CA, www.graphpad.com). Plague BTA test values were used for generating the interactive dot plots of LFA sensitivity and specificity calculations, and assay performance evaluation using MedCalc Statistical Software version 18.11.3 (MedCalc Software bvba, Ostend, Belgium; https://www.medcalc.org, 2019).

\section{Results}

A multiphase study was conducted to evaluate and assess the performance of the Plague BTA LFA; $0.15 \mathrm{~mL}$ of BTA buffer was used as negative control, and $0.15 \mathrm{~mL}$ of $Y$. pestis strain A1 122 at a concentration of $10^{6}-10^{7} \mathrm{CFU} / \mathrm{mL}$ was used as a positive control in each phase of the study. A total number of 


\section{COMPREHENSIVE EVALUATION OF ASSAY FOR DETECTION OF YERSINIA PESTIS}

Table 3. Environmental background panel

\begin{tabular}{|c|c|c|}
\hline S. No. & Organism & Strain Name \\
\hline 1 & Acinetobacter calcoaceticus & $\begin{array}{l}\text { ATCC 14987; HO-1; NBRC 12552; NCIMB 9205; CIP 66.33; DSM 1139; LMG } \\
\quad 1056\end{array}$ \\
\hline 2 & Acinetobacter haemolyticus & ATCC 17906; NCTC 10305; 2446/60; DSM 6962; CIP 64.3; NCIMB 12458 \\
\hline 3 & Acinetobacter radioresistens & ATCC 43998; DSM 6976; FO-1; CIP 103788; LMG 10613; NCIMB 12753 \\
\hline 4 & Aeromonas veronii & ATCC 35622; CDC 140-84 \\
\hline 5 & Bacillus cohnii & ATCC 51227; DSM 6307; LMG 16678 \\
\hline 6 & Bacillus horikoshii & ATCC 700161; DSM 8719; JP277; PN-121; LMG 17946 \\
\hline 7 & $\begin{array}{l}\text { Bacillus macroides (aka Lineola } \\
\quad \text { longa; Bacillus sp.) }\end{array}$ & $\begin{array}{l}\text { ATCC 12905; 1741-1b; DSM 54; NCIB 8796; NCIM 2596; NCIM 2812; LMG } \\
\quad 18474\end{array}$ \\
\hline 8 & Bacillus megaterium & $\begin{array}{l}\text { ATCC 14581; 7051; CCUG 1817, CIP 66.20, DSM 32, LMG 7127, NCIB 9376, } \\
\text { NCTC 10342, NRRL B-14308 }\end{array}$ \\
\hline 9 & Bacteroides fragilis & ATCC 23745; ICPB 3498, NCTC 10581 \\
\hline 10 & Brevundimonas diminuta & $\begin{array}{l}\text { ATCC 11568; DSM 7234; CCUG 1427, CIP 63.27, LMG 2089, NCIB 9393, NCTC } \\
\text { 8545, NRRL B-1496, USCC } 1337\end{array}$ \\
\hline 11 & Brevundimonas vesicularis & ATCC 11426; CCUG 2032, LMG 2350, NCTC 10900 \\
\hline 12 & Burkholderia cepacian & ATCC BAA-245; KC1766; LMG 16656; J2315; CCUG 48434; NCTC 13227 \\
\hline 13 & Burkholderia stabilis & $\begin{array}{l}\text { 2008724195; LMG 14294; CCUG 34168, CIP 106845, NCTC 13011; ATCC } \\
\text { BAA-67 }\end{array}$ \\
\hline 14 & Chromobacterium violaceum & ATCC 12472; NCIMB 9131; NCTC 9757; CIP 103350; DSM 30191; LMG 1267 \\
\hline 15 & Chryseobacterium gleum & ATCC 29896; CDC 3531; NCTC 10795; LMG 12451; CCUG 22176; CDC 3531 \\
\hline 16 & Chryseobacterium indologenes & ATCC 29897; CDC 3716; NCTC 10796; CCUG 14483; CIP 101026; LMG 8337 \\
\hline 17 & Citrobacter brakii & ATCC 10053 \\
\hline 18 & Citrobacter farmeri & ATCC 31897; FERM-P 5539; AST 108-1 \\
\hline 19 & Clostridium butyricum & $\begin{array}{l}\text { CDC 11875; ATCC 19398; NCTC 7423; VPI 3266; CCUG 4217; CIP 103309; } \\
\text { DSM 10702; LMG 1217; NCIMB } 7423\end{array}$ \\
\hline 20 & Clostridium perfringens & ATCC 12915; NCTC 8359; 3702/49; CIP 106516 \\
\hline 21 & Clostridium sardiniense & ATCC 33455; VPI 2971; DSM 2632; BCRC 14530 \\
\hline 22 & Comamonas testosterone & $\begin{array}{l}\text { ATCC 11996; 567201; FHP 1343; NCIMB 8955; CIP 59.24; NCTC 10698; NRRL } \\
\text { B-2611; DSM 50244; LMG 1800; CCUG } 1426\end{array}$ \\
\hline 23 & Deinococcus radiodurans & ATCC 35073; NCIMB 13156; UWO 298 \\
\hline 24 & Delftia acidovorans & ATCC 9355; LMG 1801; CCUG 1822; CIP 64.36; NCIMB 9153; NRRL B-783 \\
\hline 25 & Dermabacter hominis & ATCC 49369; DSM 7083; NCIMB 13131; CIP 105144; CCUG 32998; S69 \\
\hline 26 & Enterobacter aerogenes & $\begin{array}{l}\text { ATCC 13048; CDC 819-56; NCTC 10006; DSM 30053; CIP 60.86; LMG 2094; } \\
\text { NCIMB } 10102\end{array}$ \\
\hline 27 & Enterobacter cloacae & ATCC 10699; NCIMB 8151; CCM 1903 \\
\hline 28 & Enterococcus faecalis & ATCC 10100; NCIMB 8644; P-60 \\
\hline 29 & Escherichia coli O157:H7 & ATCC 43895; CDC EDL 933; CIP 106327; O157:H7 \\
\hline 30 & Flavobacterium mizutaii & $\begin{array}{l}\text { ATCC 33299; CIP 101122; CCUG 15907; LMG 8340; NCTC 12149; DSM 11724; } \\
\text { NCIMB 13409 }\end{array}$ \\
\hline 31 & $\begin{array}{l}\text { Fusobacterium nucleatum } \\
\text { subsp. Nucleatum }\end{array}$ & ATCC 25586; CCUG 32989; CIP 101130; DSM 15643; LMG 13131 \\
\hline 32 & Jonesia denitrificans & ATCC 14870; CIP 55.134; NCTC 10816; DSM 20603; CCUG 15532 \\
\hline
\end{tabular}


Table 3. (Continued)

\begin{tabular}{|c|c|c|}
\hline S. No. & Organism & Strain Name \\
\hline 33 & Klebsiella oxytoca & ATCC 12833; FDA PCI 114; NCDC 413-68; NCDC 4547-63 \\
\hline 34 & $\begin{array}{l}\text { Klebsiella pneumonia } \\
\text { subsp. Pneumonia }\end{array}$ & $\begin{array}{l}\text { ATCC 10031; FDA PCI 602; CDC 401-68; CIP 53.153; DSM 681; NCIMB 9111; } \\
\text { NCTC 7427; LMG } 3164\end{array}$ \\
\hline 35 & Kluyvera ascorbate & ATCC 14236; CDC 2567-61; CDC 0408-78; DSM 30109; CCUG 21164; CIP 79.53 \\
\hline 36 & Kluyvera cryocrescens & ATCC 14237; CDC 2568-61; CCUG 544; NCIMB 9139; NCTC 10484 \\
\hline 37 & Kocuria kristinae & $\begin{array}{l}\text { ATCC 27570; DSM 20032; NRRL B-14835; CCUG 33026; CIP 81.69; LMG } \\
\text { 14215; NCTC } 11038\end{array}$ \\
\hline 38 & Lactobacillus plantarum & ATCC BAA-793; LMG 9211; NCIMB 8826 \\
\hline 39 & Listeria monocytogenes & ATCC 7302; BCRC 15329 \\
\hline 40 & Microbacterium sp. & ATCC 15283; MC 100 \\
\hline 41 & Micrococcus lylae & ATCC 27566; CCUG 33027; DSM 20315; NCTC 11037; CIP 81.70; LMG 14218 \\
\hline 42 & Moraxella nonliquefaciens & $\begin{array}{l}\text { ATCC 17953; NCDC KC 770; NCTC 7784; CCUG 4863; LMG 1010; BCRC } \\
11071\end{array}$ \\
\hline 43 & Moraxella osloensis & ATCC 10973; CDC Baumann D-10; LMG 987; CCUG 34420 \\
\hline 44 & Myroides odoratus & ATCC 29979; NCTC 11179; LMG 4028; DSM 2802; CIP 105169 \\
\hline 45 & Mycobacterium smegmatis & ATCC 20; NCCB 29027 \\
\hline 46 & Neisseria lactamica & ATCC 23970; CDC A 7515; CCUG 5853; CIP 72.17; DSM 4691; NCTC 10617 \\
\hline 47 & Pseudomonas aeruginosa & ATCC 15442; NRRL B-3509; CCUG 2080; DSM 939; CIP 103467; NCIMB 10421 \\
\hline 48 & Pseudomonas fluorescens & $\begin{array}{l}\text { ATCC 13525; Migula biotype A; NCTC 10038; DSM 50090; NCIMB 9046; NRRL } \\
\text { B-2641; LMG 1794; CIP 69.13; CCUG } 1253\end{array}$ \\
\hline 49 & Ralstonia pickettii & $\begin{array}{l}\text { ATCC 27511; CCUG 3318; LMG 5942; CIP 73.23; NCTC 11149; DSM 6297; } \\
\text { NCIMB 13142; UCLA K-288 }\end{array}$ \\
\hline 50 & Rhodobacter sphaeroides & ATCC 17024; ATH 2.4 .2 \\
\hline 51 & Riemerella anatipestifer & $\begin{array}{l}\text { ATCC 11845; CCUG 14215; LMG 11054; MCCM 00568; NCTC 11014; DSM } \\
15868\end{array}$ \\
\hline 52 & $\begin{array}{l}\text { Shewanella haliotis (Pseudomo- } \\
\text { nas putrefaciens) }\end{array}$ & ATCC 49138; AmMS 201; ACM 4733 \\
\hline 53 & Shigella dysenteriae & ATCC 12039; CDC A-2050-52; NCTC 9351 \\
\hline 54 & Sphingobacterium multivorum & ATCC 33613; CDC B5533; NCTC 11343; GIFU 1347 \\
\hline 55 & Sphingobacterium spiritivorum & ATCC 33300; DSM 2582; LMG 8348 \\
\hline 56 & $\begin{array}{l}\text { Staphylococcus aureus subsp. } \\
\text { Aureus }\end{array}$ & ATCC 700699; CIP 106414; Mu 50, MRSA \\
\hline 57 & Staphylococcus capitis & ATCC 146; NRRL B-2616; BCRC 15248 \\
\hline 58 & Stenotrophomonas maltophilia & $\begin{array}{l}\text { ATCC 13637; NCIMB 9203; NCTC 10257; NRC 729; CIP 60.77; DSM 50170; } \\
\text { LMG 958; NRRL B-2756 }\end{array}$ \\
\hline 59 & Streptococcus equinus & ATCC 15351; 7H4; NBRC 12057; IFO 12057 \\
\hline 60 & Streptomyces coelicolor & ATCC 10147; DSM 41007; NIHJ 147; NBRC 3176 \\
\hline 61 & Vibrio cholerae & ATCC 14104; BG29 \\
\hline
\end{tabular}

1,182 LFA tests were performed in this study, and details about positive and negative controls tested and number of samples tested in each phase of the evaluation are shown in Table 5.

In the Phase 1 range finding and reproducibility study, a total of 185 tests were performed: 35 tests for range-finding of strain CO99-3015, 30 tests for range-finding of strain A1122, and 120 tests for reproducibility LOD testing of strain CO99-3015, along with positive and negative controls. All samples tested at a concentration $\geq 10^{5}$ to $10^{6} \mathrm{CFU} / \mathrm{mL}$ were positive. In Phase 2 inclusivity panel evaluation, a total of 
Table 4. White Powder Panel

\begin{tabular}{|c|c|c|}
\hline S.No. & Material & Source \\
\hline 1 & Dipel (Bacillus thuringiensis) & Summerwinds Nursery, Palo Alto, VA \\
\hline 2 & Powdered milk & Raley's Grocery Store, Pleasanton, CA \\
\hline 3 & Powdered coffee creamer & Raley's Grocery Store, Pleasanton, CA \\
\hline 4 & Powdered sugar & Raley's Grocery Store, Pleasanton, CA \\
\hline 5 & Talcum powder & Raley's Grocery Store, Pleasanton, CA \\
\hline 6 & Wheat flour & Van's, Livermore, CA \\
\hline 7 & Soy flour & Van's, Livermore, CA \\
\hline 8 & Rice flour & Ranch 99, Pleasanton, CA \\
\hline 9 & Baking soda & Target Stores, Livermore, CA \\
\hline 10 & Chalk dust & Target Stores, Livermore, CA \\
\hline 11 & Brewer's yeast & GNC Stores, Livermore, CA \\
\hline 12 & Drywall dust & Home Depot, Livermore, CA \\
\hline 13 & Cornstarch & Raley's Grocery Store, Pleasanton, CA \\
\hline 14 & Baking powder & Raley's Grocery Store, Pleasanton, CA \\
\hline 15 & GABA (Gama aminobutyric acid) & Sigma-Aldrich Corp, St. Louis, MO \\
\hline 16 & L-Glutamic acid & Sigma-Aldrich Corp, St. Louis, MO \\
\hline 17 & Kaolin & Sigma-Aldrich Corp, St. Louis, MO \\
\hline 18 & Chitin & Sigma-Aldrich Corp, St. Louis, MO \\
\hline 19 & Chitosan & Sigma-Aldrich Corp, St. Louis, MO \\
\hline 20 & Magnesium sulfate $(\mathrm{MgSO} 4)$ & Sigma-Aldrich Corp, St. Louis, MO \\
\hline 21 & Boric acid & Sigma-Aldrich Corp, St. Louis, MO \\
\hline 22 & Powdered toothpaste & Walmart Pharmacy, Livermore, CA \\
\hline 23 & Popcorn salt & Raley's Grocery Store, Pleasanton, CA \\
\hline 24 & Baby powder & Target Stores, Livermore, CA \\
\hline 25 & Powdered infant formula, iron fortified & Target Stores, Livermore, CA \\
\hline 26 & Powdered infant formula, low iron & Target Stores, Livermore, CA \\
\hline
\end{tabular}

Table 5. Details of the number of samples tested, including the positive and negative controls by plague BTA LFA test in each of the 7 phases

\begin{tabular}{|l|c|c|c|c|c|}
\hline Yp LFA Test Phases & $\begin{array}{c}\text { Positive } \\
\text { Control }\end{array}$ & $\begin{array}{c}\text { Negative } \\
\text { Control }\end{array}$ & $\begin{array}{c}\text { Positive } \\
\text { Test Result }\end{array}$ & $\begin{array}{c}\text { Negative } \\
\text { Test Result }\end{array}$ & Total \\
\hline $\begin{array}{l}\text { Phase 1: Linear dynamic range and reproducibility } \\
\text { testing }\end{array}$ & 5 & 11 & 164 & 21 & 201 \\
\hline Phase 2: Inclusivity panel testing & 5 & 5 & 80 & 5 & 100 \\
\hline Phase 3: Near-neighbor panel testing & 5 & 5 & 0 & 305 & 315 \\
\hline $\begin{array}{l}\text { Phase 4: Environmental background microbes } \\
\text { testing }\end{array}$ & 5 & 5 & 0 & 130 & 145 \\
\hline $\begin{array}{l}\text { Phase 5a: White powder panel testing } \\
\text { Phase 5b: White powder panel spike testing }\end{array}$ & 5 & 5 & 10 & 50 & 140 \\
\hline Phase 6: Environmental aerosol filter extract testing & 3 & 3 & 5 & 5 & 16 \\
\hline $\begin{array}{l}\text { Phase 7: Temperature dependent expression of F1 } \\
\text { capsular antigen panel testing }\end{array}$ & 0 & 0 & 80 & 80 & 160 \\
\hline Total & 38 & 39 & 459 & 646 & 1,182 \\
\hline
\end{tabular}


90 tests (5 replicates of 18 different $Y$. pestis strains) were tested. Near neighbors of $Y$. pestis were evaluated in Phase 3, and a total of 100 tests ( 5 replicates of 20 near neighbor strains along with positive and negative controls) were performed. In the Phase 4 environmental panel evaluation, a total of 305 tests 5 replicates of 61 different bacterial strains) were tested. In the Phase 5 white powder and environmental aerosol sample test evaluation, a combined total of 260 tests (5 replicates of 26 white powders) in the absence and presence of $Y$. pestis A1122 were performed. In Phase 6, BioWatch aerosol filter extract was tested in 5 replicates each, with and without the spiking of $Y$. pestis A1122. In phase 7 , the temperature-dependent detection of F1 capsular antigen was evaluated with a total of 160 tests, 20 replicates each of 4 different strains grown at $25^{\circ} \mathrm{C}$ or $35^{\circ} \mathrm{C}$.

Data from Phase 1 range-finding study, 35 tests $(5$ replicates for each of 7 different concentrations) of $Y p$ strain CO99-3015 and 30 tests (6 replicates of 5 different concentrations) of $Y p$ strain A1122 were used in the Probit regression analysis for determining the LOD of Plague BTA LFA visual results. LOD was calculated as the concentration that corresponds to a probability of 0.95 , which is equivalent to the estimated LOD within $95 \%$ confidence intervals. ${ }^{27}$ Probit analysis curves are shown in Figure 1 for estimating the LOD for $Y$. pestis strains CO99-3015 and A1122. The calculated LOD based on Phase 1 data and Probit analysis for $Y$. pestis C099-3015 was $2.3 \times 10^{5} \mathrm{CFU} / \mathrm{mL}$ (3.4 x $10^{4} \mathrm{CFU} /$ assay), and for $Y$. pestis A1122, it was $4.4 \times 10^{4}$ $\mathrm{CFU} / \mathrm{mL}\left(6.6 \times 10^{3} \mathrm{CFU} /\right.$ assay $)$.
BTA Reader values were plotted against various concentrations of $Y$. pestis for determining the limit of detection as shown in Figure 2. The curves show that $10^{5}-10^{6}$ $\mathrm{CFU} / \mathrm{mL}$ uniformly gave positive results above the BTA Reader cut-off value of 200 and determined as the LOD.

The Plague LFA assay was further tested in Phase 1 for repeatability by 2 operators, using a final concentration of $10^{5}-10^{6}$ for a total of 120 tests using $Y$. pestis strain CO99-3015, of which 120 of 120 tests gave expected positive results (both by visual observation and by BTA Reader call).

In Phase 2, an inclusivity panel of $18 Y$. pestis strains was tested 5 times each by 1 of 2 different operators (Table 1 ). The inclusivity panel consisted of 17 F1 capsular antigen positive strains and one F1 capsular antigen minus strain, Angola O.PE3. The $17 \mathrm{~F} 1$ capsular antigen positive $Y$. pestis strains yielded 85 positive visual results and 84 of 85 BTA Reader positives. The 1 BTA Reader negative plague LFA was re-read on a second reader and was positive. The $1 \mathrm{~F} 1$ capsular antigen minus strain yielded 5 negative visual results and 5 negative BTA Reader results.

In Phase 3, a near neighbor panel of 20 Yersinia (nonpestis) strains (Table 2) was tested by 5 different operators at a concentration of $10^{8}$ to $10^{9} \mathrm{CFU} / \mathrm{mL}$ (3 logs above the LOD). All tests yielded negative results both visually and by BTA Reader. In Phase 4, an environmental panel of 61 strains (Table 3) yielded negative test results for 58 of 61 (95\%) strains by visual observation and using BTA Reader. Positive plague LFA results were observed with Brevundimonas

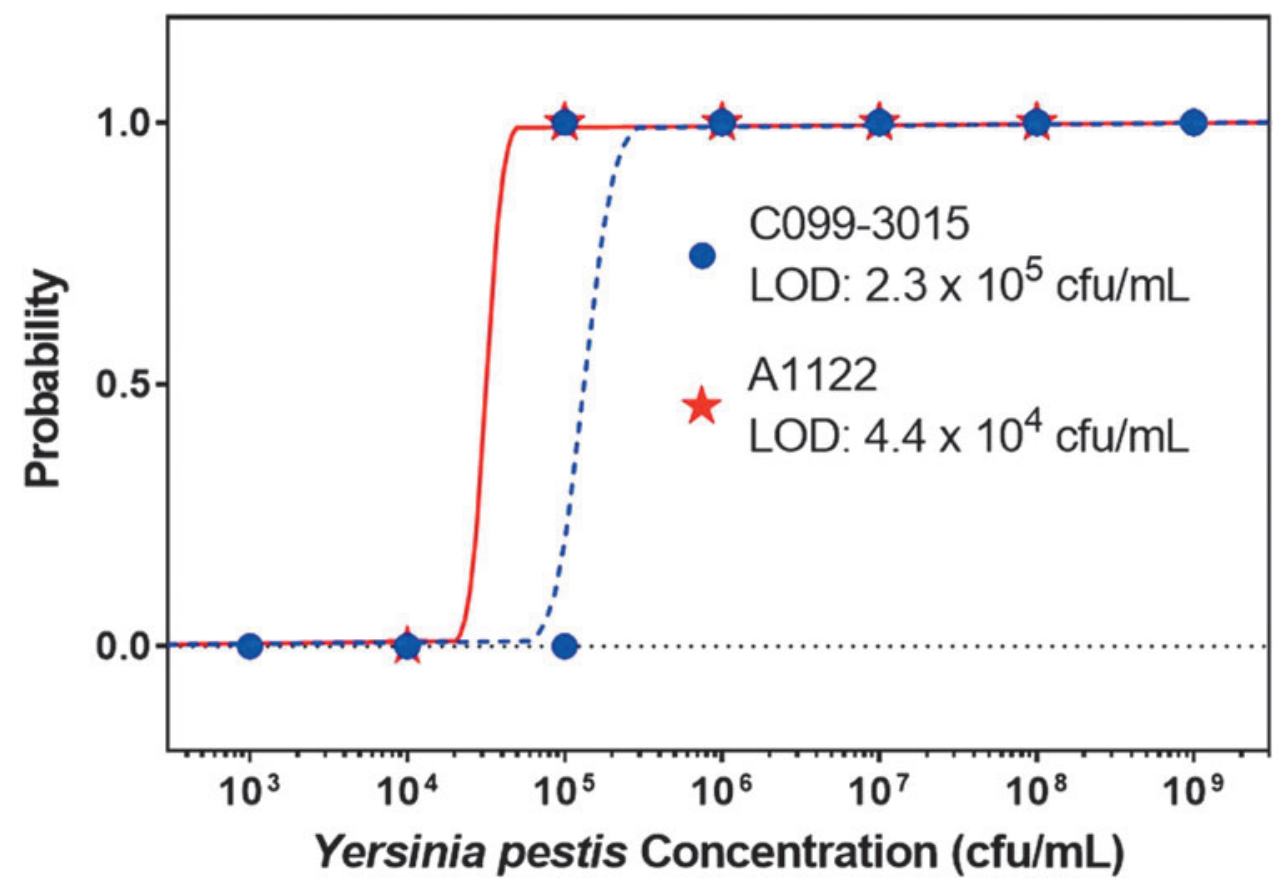

Figure 1. Probit regressions for the $Y$. pestis strains C099-3015 and A1122 strains are shown as 2 different lines in the scatter plot. The curves are drawn using the calculated probability of detection as a function of spore concentration. Limit of detection of the Yp LFA test was estimated by finding the $Y$. pestis strain concentration with a probability of detection at 0.95 . For $Y$. pestis C099-3015, the LOD is $2.3 \times 10^{5} \mathrm{CFU} / \mathrm{mL}$, and for $Y$. pestis A1122 the LOD is $4.4 \times 10^{4} \mathrm{CFU} / \mathrm{mL}$. 
diminuta, Myroides odoratus, and Staphylococcus aureus. In Phase 5, the white powders did not affect the performance of the LFA as evidenced by the presence of the positive control line in 130 of 130 tested samples and the absence of any false-positive result in the test line. When the powders were spiked with $Y$. pestis strain A1122, positive results were observed with 125 of 130 tests. Inhibition (5 of 130 tests) of detection of $Y$. pestis strain A1122 was observed with only chalk dust and drywall dust. In Phase 6 , the environmental filter extract did not inhibit the performance of the LFA, as evidenced by the presence of a positive control line in 5 of 5 tested samples without falsepositive result. Filter extract spiked with $Y$. pestis strain A1122 yielded positive results in all 5 replicates.

The Plague LFA assay was also evaluated in Phase 7 for its ability to detect temperature-dependent expression of $Y$. pestis F1 capsular antigen. Four $Y$. pestis strains representing each of the biovars C099-3015 (Orientalis), Nepal 516 (Antiqua), PyH1R3 (Mediaevalis), and Pestoides A (Pestoides), were tested 20 times each for the 2 growth conditions (a total of 160 tests were performed). Strains grown at $25^{\circ} \mathrm{C}$ resulted in 80 of 80 visual negative results, and 73 of 80 negative BTA reads. The 7 positive LFA strips were re-read on a second BTA reader and were negative. Strains grown at $35^{\circ} \mathrm{C}$ for 24 hours followed by $25^{\circ} \mathrm{C}$ for 4 days resulted in $100 \%$ positive results both visually and by the BTA Reader. The effect of growth temperature on detection was statistically significant $(P<0.0001)$ (Figure 3$)$.

Excluding the 33 positive controls and 39 negative controls performed during the evaluation, the data from the
1,110 tests performed during Phases 1 through 7 were used to calculate sensitivity and specificity. Sensitivity, specificity, and accuracy are basic measures of performance for a diagnostic/detection test. Together, they describe how well the test can determine whether the $Y$. pestis $\mathrm{F} 1$ antigen (the analyte) is present or absent in the tested sample. Data from visual reads of the plague BTA LFA are displayed in a $2 \times 2$ contingency table format (Table 6). Test results fall in 1 of the 4 categories: true positive (TP, $Y$. pestis antigen present, and test positive); false positive (FP, $Y$. pestis antigen not present but test positive); false negative (FN, $Y$. pestis antigen present but test negative), and true negative ( $Y$. pestis antigen absent and test negative). Of the 1,110 tests performed, 460 were true positive, 625 were true negative, 15 were false positive, and 10 were false negative.

Sensitivity is defined as the proportion of true positives that are correctly identified by the test:

$$
\text { Sensitivity }(\%)=100 \times \frac{T P}{T P+F N}
$$

Specificity is defined as the proportion of true negatives that are correctly identified by the test and is calculated as:

$$
\text { Specificity }(\%)=100 \times \frac{T N}{T N+F P} \text {. }
$$

Accuracy is defined as the proportion of the total number of true-positive and true-negative tests that are correctly identified by the test and is calculated as:

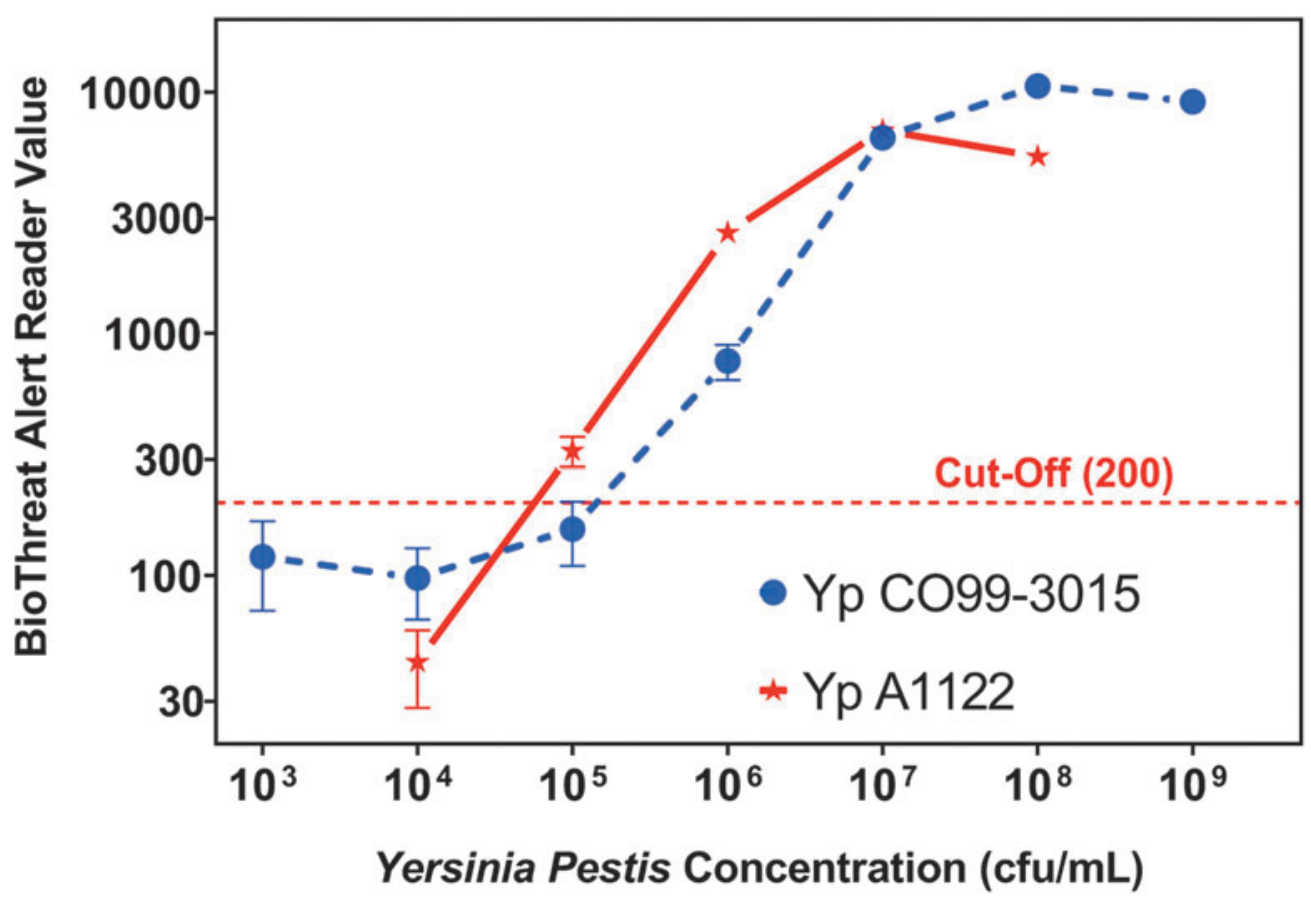

Figure 2. The titration curves depict BTA reader value with respect to the concentration of $Y$. pestis strains C099-3015 and A1122. The curves were generated using the average of at least 5 replicates, and the error bars are the standard deviations. The cut-off value of 200 is shown as a dashed line. For both strains, the first test concentration that is above the cut-off value is $10^{5} \mathrm{CFU} / \mathrm{mL}$. 


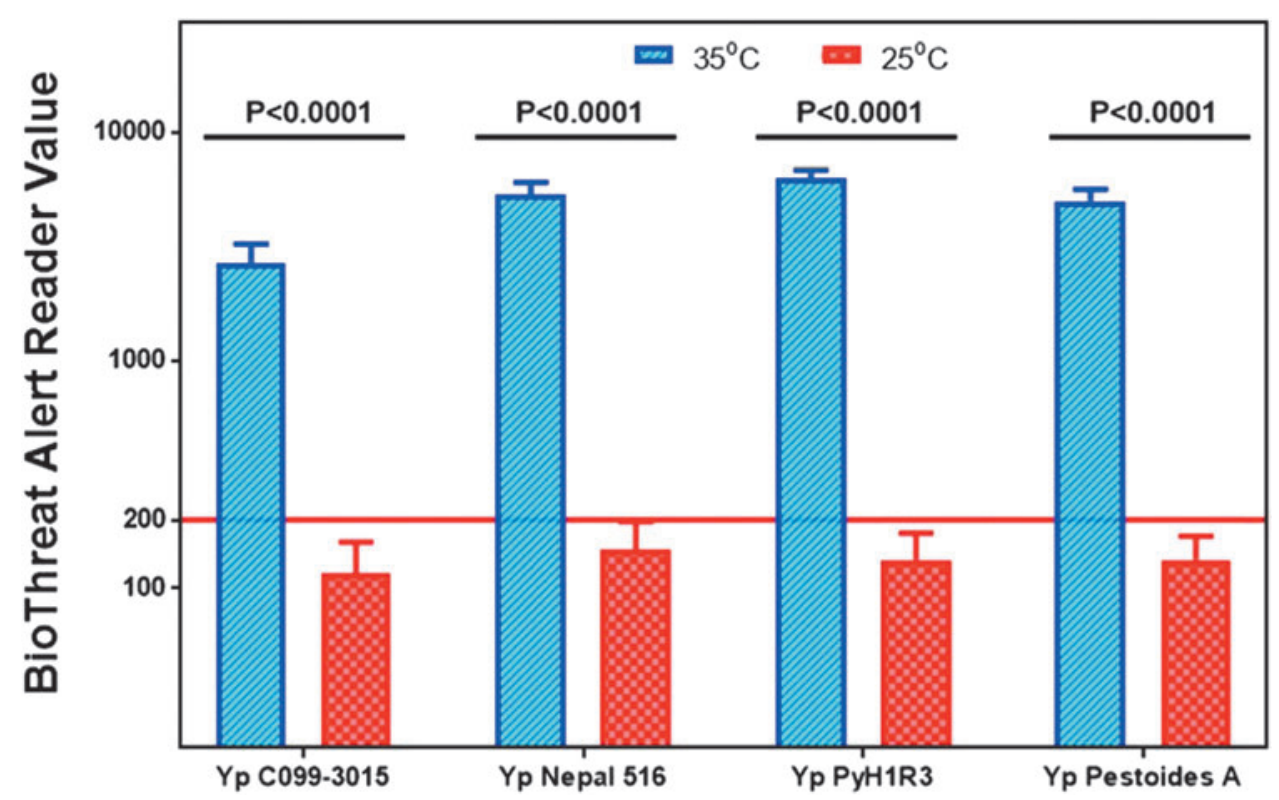

Figure 3. Bar diagram that summarizes the testing performed on $Y$. pestis strains Yp, C099-3015 (Orientalis); Yp, Nepal 516 (Antiqua); Yp, PyH1R3 (Mediaevalis); Yp, Pestoides A (Pestoides) were grown either at $25^{\circ} \mathrm{C}$ or at $35^{\circ} \mathrm{C}$, or 24 hours followed by $25^{\circ} \mathrm{C}$ for 4 additional days. It provides a visual representation of the BTA Reader values in 2 different growth temperatures. The number of tests performed per sample are displayed at the top of each cluster. The cutoff value of 200 is shown as a solid line. Any data points that were above the cut-off value are positive, while any data points below the cut-off value are negative.

$$
\operatorname{Accuracy}(\%)=100 \times \frac{T N+T P}{T P+F N+T N+F P} .
$$

Data from only $1,110 Y p$ BTA LFA test results are used for calculations. Data from testing of 72 samples that were used as positive and negative controls were excluded in the diagnostic test sensitivity and specificity determination. Table 6 shows the $2 \times 2$ contingency table and statistical analysis results for the resulting sensitivity $(97.868 \%)$, specificity $(97.660 \%)$, and accuracy $(97.748 \%)$ of this assay.

To further evaluate the assay, the BTA Reader values were used to generate a Receiver Operating Characteristic (ROC) curve. For Phases 1, 2, and 7, the BTA Reader values used were from the rerun on the second reader. Even though the reader values are not quantitative, the values can be used to further evaluate the accuracy of a detection test to discriminate the test positive samples from those that are test negative using ROC analysis. The sensitivity and specificity were calculated for every possible cut-off point selected to discriminate between the positive and negative populations. This curve was created by plotting the true-positive rate as a function of the false-negative rate for every possible cut-off point. Figure 4 shows the ROC curve for the Plague LFA, having the area under the curve 0.99 , thus indicating that this test is highly specific and sensitive. The sensitivity $(97.65 \%)$ and specificity $(96.57 \%)$ calculated from the ROC curve are based on a BTA cut-off

Table 6. 2 × 2 Contingency table to assess the accuracy of $Y$. pestis LFA by visual read

\begin{tabular}{|l|c|c|c|}
\hline Plague BTA LFA & Y. pestis Positive & Y. pestis Negative & Total \\
\hline Test Positive & 459 & 15 & 674 \\
\hline Test Negative & 10 & 626 & 1,110 \\
\hline Total & 469 & 641 & Confidence Interval \\
\hline \multicolumn{2}{|c|}{ Statistical analysis of Y. pestis LFA performance } \\
\hline Parameter & Percentage & $96.114 \%$ to $98.973 \%$ \\
\hline Sensitivity & $97.868 \%$ & $96.170 \%$ to $98.684 \%$ \\
\hline Specificity & $97.660 \%$ & 0.967 to 0.986 \\
\hline Area Under the Curve (AUC) & 0.978 & \\
\hline Accuracy & $97.748 \%$ & \\
\hline
\end{tabular}




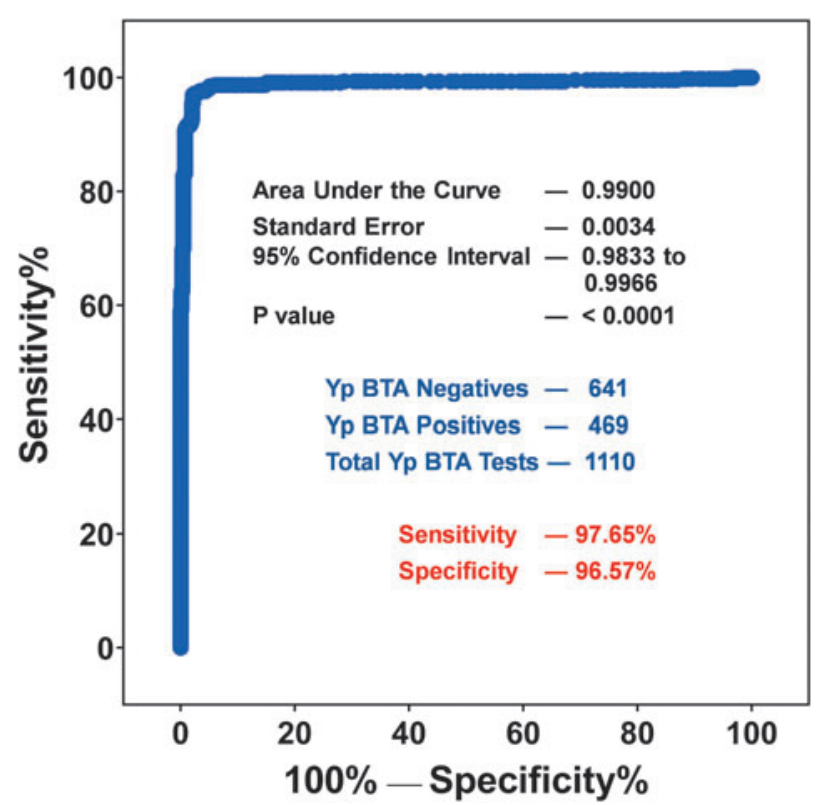

Figure 4. Receiver operator characteristic (ROC) curve provides a graphic representation of the sensitivity and specificity of the visual test results of $Y p$ LFA test. Each point on the curve is a possible cut-off value, and its place on the curve is determined by its specificity and sensitivity. The calculated area under the curve (AUR) was 0.99, thus indicating that the assay is accurate and reliable.

value of 200 . These values are lower than the calculated sensitivity $(97.868 \%)$ and specificity $(97.660 \%)$ shown using visual plague BTA LFA results (Table 6). The Youden index $\mathrm{J}$ is the maximum vertical distance between the ROC curve and the line of equality. The cutoff value that responds to the Youden index J can give the optimal combination of sensitivity and specificity, if the disease prevalence is $50 \%$. The Youden index $\mathrm{J}$ calculated from this ROC curve was 0.9483 , and the calculated best sensitivity of $97.01 \%$ and specificity of $97.82 \%$ at a cut-off of BTA Reader value $>238$. When reader value is available, different cut-offs can be set to calculate the sensitivity and specificity of the assay.

In addition, data required for ROC analysis can also be depicted as an interactive dot plot (Figure 5) for measuring the sensitivity and specificity. In this plot, the reader values are shown on the $Y$ axis, and different cut-off values can be used to estimate the sensitivity and specificity at that value. In this analysis, a threshold reader value of 200 also gave a sensitivity of $97.65 \%$ and specificity of $96.57 \%$.

\section{Discussion}

In addition to natural outbreaks (ie, the 3 major pandemics), $Y$. pestis has been employed and/or developed as a biological weapon. During World War II, Japan's in-

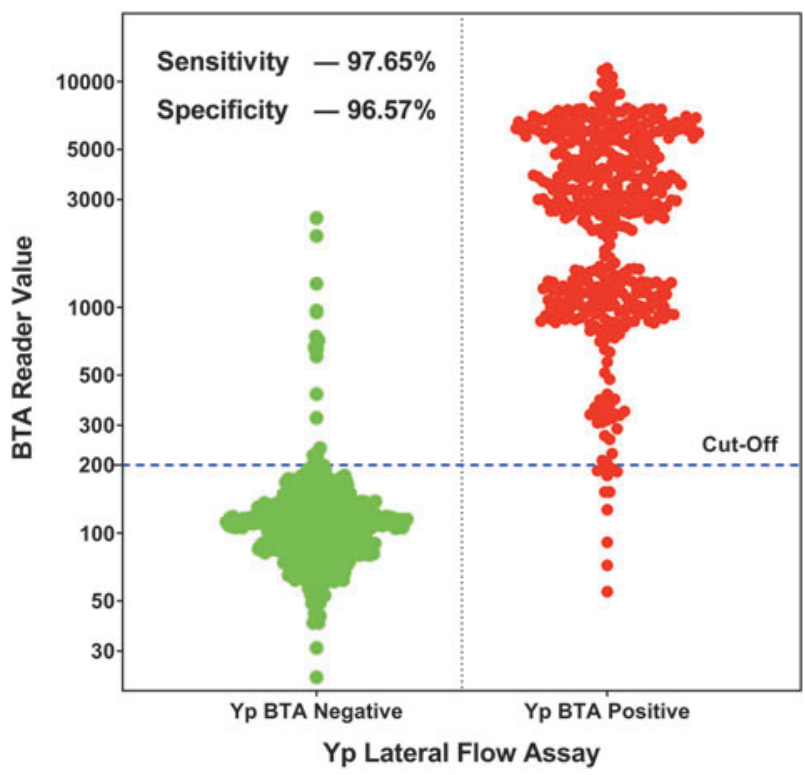

Figure 5. A dot density diagram that shows all 1,110 tests performed grouped as designated positive and designated negative by the BTA Reader. The cut-value of 200 is shown as a solid line. The calculated assay sensitivity is $97.65 \%$, and the specificity is $96.57 \%$. Any data points in the designated negative group that were above the cut-off value are false positive, while any data points in the designated positive group that were below the cutoff value are false negative.

famous Unit 731 purportedly dropped plague-infected fleas over Manchuria. ${ }^{4}$ Both the United States and the former Soviet Union investigated the use of $Y$. pestis as a weapon, including methods of aerosolization allowing direct dispersal of the bacteria. ${ }^{4,7,14}$

An effective public health response to $Y$. pestis involves several facets, including use of medical countermeasures (vaccines, antimicrobials) and methods for surveillance and early diagnosis. Because of the high mortality rates associated with untreated pneumonic plague, rapid identification of a deliberate release is critical so that medical countermeasures can be rapidly deployed and potentially exposed individuals can be treated. Currently, many technologies exist for the detection and identification of $Y$. pestis. Polymerase chain reaction (PCR) assays and platforms, including the RAPID and SmartCycler, have demonstrated sensitivities of $50 \mathrm{fg}$ DNA $(\sim 10$ genomic equivalents, GE) for single-probe assays, ${ }^{28}$ while the FilmArray detected $Y$. pestis strains at 250 to $25,000 \mathrm{GE} .^{29}$ Yang et $\mathrm{al}^{30}$ investigated the use of 2 suspension arrays for the detection of $Y$. pestis, one of which could identify only to the genus level. The species-specific assay had a detection limit of $50 \mathrm{fg}$ DNA. Fluorescence in situ hybridization has also been investigated but is impractical because of its lengthy assay time ( $\sim 8$ hours to results). ${ }^{32}$ Most antibody-based assays target the F1 capsular protein. ${ }^{33-36}$

LFA assays were first commercially introduced for pregnancy testing in $1988 .{ }^{37}$ LFA assays require minimum 
sample and no specialized equipment ${ }^{38}$ and could be used by first responders and law enforcement officers to test suspicious materials in field settings. An F1-based lateral flow that used monoclonal antibodies to F1 has been developed and used in Madagascar to test bubo aspirates from suspected plague patients. ${ }^{32} \mathrm{~F} 1$ antigen is temperature regulated and not expressed at temperatures $<33^{\circ} \mathrm{C}$; as an alternative, a dipstick employing antibodies against the Pla protein is not limited by variations in growth temperature, although it is present in $Y$. pseudotuberculosis and $Y$. enterocolitica, and recent studies have demonstrated it is also found in other bacteria and therefore not a specific to $Y$. pestis. ${ }^{33}$ Anti-LPS antibodies have also been evaluated and may hold promise for species-specific detection. ${ }^{34}$

Other BTA LFA assays have previously been evaluated for the detection of biothreat agents, including orthopoxviruses, ${ }^{39}$ ricin, ${ }^{40}$ abrin, ${ }^{41}$ and Bacillus anthracis. ${ }^{25}$ Limited evaluations have also been conducted with assays for the detection of Francisella tularensis (unpublished data), botulinum neurotoxins, ${ }^{42}$ and staphylococcal enterotoxins. ${ }^{43}$ The Plague BTA LFA test uses a combination of a polyclonal capture antibody and monoclonal detect antibody to selectively capture and detect the presence of F1 antigen in aqueous samples. The purpose of the current study was to evaluate the performance of the Plague LFA assay in order to understand its sensitivity, specificity, reproducibility, and limitations for potential use in the field. Using the BTA Reader and the manufacturer's recommended cutoff of 200, we estimated the LOD of the Plague LFA to be approximately $10^{5}$ to $10^{6} \mathrm{CFU} / \mathrm{mL}$. This LOD is lower than reported in an earlier study, in which Zasada et al demonstrated an LOD of $10^{7} \mathrm{CFU} / \mathrm{mL}$ for $Y$. pestis using the Plague LFA assay. The difference in LOD may be because in the previous study, $Y$. pestis organisms were inactivated by heating at $60^{\circ} \mathrm{C}$ for 22 hours prior to testing with the Plague BTA Test Strip. Limitations of the Plague BTA LFA include the relatively high LOD compared to real-time PCR methods and the inability to detect $Y$. pestis grown at $25^{\circ} \mathrm{C}$.

To evaluate the sensitivity and specificity of the Plague BTA LFA assay, $18 Y$. pestis strains belonging to 4 different biovars and of different geographic origins were used. Seventeen of these strains yielded positive results visually when tested at a concentration 2 logs above the LOD. The Angola O.PE3 strain is an F1 capsular antigen minus strain and, as expected, was negative by this assay. The Plague LFA was also tested against 20 Yersinia near neighbors as well as 61 other organisms commonly encountered in the environment. Positive results were observed with environmental background strains; $B$. diminuta, $M$. odoratus (the F1 capsule antibody used in the Tetracore Plague LFA targets an antigenic epitope that is also present in $M$. odoratus and $B$. diminuta where there is an overlap in the F1 capsule antigen protein sequence from positions 103 to 119 ), and S. aureus. Antibodies used in the Plague BTA LFA are purified on a protein A column, and this protein is found in the cell walls of some organisms (eg, S. aureus).
In this study, we evaluated the ability of this assay to detect $Y$. pestis in the presence of commonly encountered powders and extracts taken from environmental filters. The Plague LFA yielded positive results in 24 of 26 powders spiked with $Y$. pestis A1122 at a final concentration of $10^{6}-10^{7} \mathrm{CFU} / \mathrm{mL}$. Inconsistent results were observed with chalk dust and drywall dust, both of which consist of relatively large particles that may inhibit the flow of fluid across the test strip and that may also act as a filter to prevent the antigen from interacting with the antibody, resulting in a false-negative result. Pooled environmental filter extracts alone yielded negative results and did not prevent the assay from detecting $Y$. pestis at a final concentration of $10^{6}-10^{7} \mathrm{CFU} / \mathrm{mL}$.

Variability in readings between BTA Readers was encountered, which yielded either false-positive or falsenegative readings. In the case of false-positive results, the reader typically produced values near the cut-off. These findings highlight the importance of these assays being performed by trained and experienced users with an understanding of the limitations of sample testing and result interpretation.

In conclusion, the results presented here demonstrate a sensitivity (97.87\%), specificity (97.66\%), accuracy (97.75\%), and limit of detection $\left(10^{4}-10^{5} \mathrm{CFU} / \mathrm{ml}\right)$ for the Plague BTA LFA. These performance data are important for accurate interpretation of qualitative results arising from testing suspicious white powders and aerosol samples in the field. It should be noted that specificity of this test has not been evaluated for other environmental specimens such as soil or water. It is not approved for human clinical diagnostic use. Highly suspicious samples should be tested by other methods in a reference laboratory. If only limited or very sparse material is available, it should be collected and submitted to a CDC LRN laboratory for testing. In addition, this assay is based on detection of $Y$. pestis $\mathrm{F} 1$ capsule, an antigen expressed only at $\geq 33^{\circ} \mathrm{C}$. Any positive specimen in this assay is considered presumptive positive and should be referred to the CDC LRN for additional testing, confirmation, and characterization for an appropriate public health response.

\section{REFERENCES}

1. Amedei A, Niccolai E, Marino L, D'Elios MM. Role of immune response in Yersinia pestis infection. I Infect Dev Ctries 2011;5(9):628-639.

2. Balali-Mood M, Moshiri M, Etemad L. Medical aspects of bio-terrorism. Toxicon 2013;69:131-142.

3. Gage KL, Dennis DT, Tsai TF. Prevention of plague: recommendations of the Advisory Committee on Immunization Practices (ACIP). MMWR Recomm Rep 1996;45(RR-14): $1-15$.

4. Inglesby TV, Dennis DT, Henderson DA, et al. Plague as a biological weapon: medical and public health management. Working Group on Civilian Biodefense. JAMA 2000;283(17): 2281-2290. 
5. Mikula KM, Kolodziejzyk R, Goldman A. Yersinia infection tools - characterization of structure and function of adhesins. Front Cell Infect Microbiol 2013;2:169.

6. Perry RD, Fetherston JD. Yersinia pestis—etiologic agent of plague. Clin Microbiol Rev 1997;10(1):35-66.

7. Riedel S. Plague: from natural disease to bioterrorism. Proc (Bayl Univ Med Cent) 2005;18(2):116-124.

8. Cui Y, Yu C, Yan Y, et al. Historical variations in mutation rate in an epidemic pathogen, Yersinia pestis. Proc Nat Acad Sci U S A 2013;110(2):577-582.

9. Drancourt M. Plague in the genomic area. Clin Microbiol Infect 2012;18(3):224-230.

10. Butler T. Plague gives surprises in the first decade of the $21 \mathrm{st}$ century in the United States and worldwide. Am J Trop Med Hyg 2013;89(4):788-793.

11. Frean J, Klugman KP, Arntzen L, Bukofzer S. Susceptibility of Yersinia pestis to novel and conventional antimicrobial agents. J Antimicrob Chemother 2003;52(2):294-296.

12. Byvalov AA, Ovodov YS. Immunobiological properties of Yersinia pestis antigens [in Russion]. Bioorg Khim 2011;37(4): 452-463.

13. Chen PE, Cook C, Stewart AC, et al. Genomic characterization of the Yersinia genus. Genome Biol 2010; 11(1):R1.

14. Agar SL, Sha J, Foltz SM, et al. Characterization of a mouse model of plague after aerosolization of Yersinia pestis CO92. Microbiology 2008;154(Pt 7):1939-1948.

15. Galimand M, Guiyoule A, Gerbaud G, et al. Multidrug resistance in Yersinia pestis mediated by a transferable plasmid. N Engl J Med 1997;337(10):677-680.

16. Cornelius C, Quenee L, Anderson D, Schneewind O. Protective immunity against plague. In: Perry RD, Fetherston JD, eds. The Genus Yersinia. New York: Springer; 2007:415-424.

17. Derewenda U, Mateja A, Devedjiev Y, et al. The structure of Yersinia pestis $\mathrm{V}$-antigen, an essential virulence factor and mediator of immunity against plague. Structure 2004;12(2): 301-306.

18. Kolodziejek AM, Schnider DR, Rohde HN, et al. Outer membrane protein X (Ail) contributes to Yersinia pestis virulence in pneumonic plague and its activity is dependent on the lipopolysaccharide core length. Infect Immun 2010;78(12): 5233-5243.

19. Leal-Balbino TC, Leal NC, Lopes CV, de Almeida AMP. Differences in the stability of the plasmids of Yersinia pestis cultures in vitro: impact on virulence. Mem Inst Oswaldo Cruz 2004;99(7):727-732.

20. Grosfeld H, Cohen S, Bino T, et al. Effective protective immunity to Yersinia pestis infection conferred by DNA vaccine coding for derivatives of the F1 capsular antigen. Infect Immun 2003;71(1):374-383.

21. Sebbane F, Jarrett CO, Gardner D, Long D, Hinnebusch BJ. Role of the Yersinia pestis plasminogen activator in the incidence of distinct septicemic and bubonic forms of fleaborne plague. Proc Nat Acad Sci U S A 2006;103(14):55265530 .

22. Haensch S, Bianucci R, Signoli M, et al. Distinct clones of Yersinia pestis caused the Black Death. PLoS Pathog 2010; 6(10):e1001134.

23. Price PA, Jin J, Goldman WE. Pulmonary infection by Yersinia pestis rapidly establishes a permissive environment for microbial proliferation. Proc Nat Acad Sci U S A 2012; 109(8):3083-3088.

24. Centers for Disease Control and Prevention. Biosafety in Microbiological and Biomedical Laboratories. 5th ed. 2009. https://www.cdc.gov/labs/BMBL.html. Accessed October 29, 2019.

25. Ramage JG, Prentice KW, DePalma L, et al. Comprehensive laboratory evaluation of a highly specific lateral flow assay for the presumptive identification of Bacillus anthracis spores in suspicious white powders and environmental samples. Health Secur 2016;14(5):351-365.

26. Standard method performance requirements for immunological-based handheld assays (HHAs) for the detection of Bacillus anthracis spores in visible powders. AOAC SMPR 2010.004: J AOAC Int 2011;94(4):1352-1355.

27. Clinical and Laboratory Standards Institute. Evaluation of detection capability for clinical laboratory measurement procedures; Approved guideline-Second Edition. CLSI document EP17-A2. Wayne, PA: Clinical and Laboratory Standards Institute; 2012.

28. Christensen DR, Hartman LJ, Loveless BM, et al. Detection of biological threat agents by Real-Time PCR: comparison of assay performance on the R.A.P.I.D., the Light Cycler, and the Smart Cycler platforms. Clin Chem 2006;52(1): 141-145.

29. Seiner DR, Colburn HA, Baird C, et al. Evaluation of the FilmArray ${ }^{\circledR}$ system for detection of Bacillus anthracis, Francisella tularensis and Yersinia pestis. J Appl Microbiol 2013;114(4): 992-1000.

30. Yang Y, Wang J, Wen H, Liu H. Comparison of two suspension arrays for simultaneous detection of five biothreat bacterial in powder samples. J Biomed Biotechnol 2012;2012:831052.

31. Loïez C, Herwegh S, Wallet F, Armand S, Guinet F, Courcol RJ. Detection of Yersinia pestis in sputum by realtime PCR. J Clin Microbiol 2003;41(10):4873-4875.

32. Kenny JH, Zhou Y, Schriefer ME, Bearden SW. Detection of viable Yersinia pestis by fluorescence in situ hybridization using peptide nucleic acid probes. J Microbiol Methods 2008; 75(2):293-301.

33. Chanteau S, Rahalison L, Ralafiarisoa L, et al. Development and testing of a rapid diagnostic test for bubonic and pneumonic plague. Lancet 2003;361(9353):211-216.

34. Simon S, Demeure C, Lamourette P, et al. Fast and simple detection of Yersinia pestis applicable to field investigation of plague foci. PLoS One 2013;8(1):e54947.

35. Anish C, Guo X, Wahlbrink A, Seeberger PH. Plague detection by anti-carbohydrate antibodies. Angew Chem Int Ed Engl 2013;52(36):9524-9528.

36. Zhao T, Zhao P, Doyle MP. Detection and isolation of Yersinia pestis without fraction 1 antigen by monoclonal antibody in foods and water. J Food Protect 2012;75(9):1555-1561.

37. Gubala V, Harris LF, Ricco AJ, Tan MX, Williams DE. Point of care diagnostics: status and future. Anal Chem 2012; 84(2):487-515.

38. Andreotti PE, Ludwig GV, Peruski AH, Tuite JJ, Morse S, Peruski LF Jr. Immunoassay of infectious agents. Biotechniques 2003;35(4):850-859.

39. Townsend MB, MacNeil A, Reynolds MG, et al. Evaluation of the Tetracore Orthopox BioThreat ${ }^{\circledR}$ antigen detection assay using laboratory grown orthopoxviruses and rash illness clinical specimens. J Virol Methods 2013;187:37-42. 
40. Hodge DR, Prentice KW, Ramage JG, et al. Comprehensive laboratory evaluation of a highly specific lateral flow assay for the presumptive identification of ricin in suspicious white powders and environmental samples. Biosecur Bioterror 2013; 11(4):237-250.

41. Ramage JG, Prentice KW, Morse SA, et al. Comprehensive laboratory evaluation of a specific lateral flow assay for the presumptive identification of abrin in suspicious white powders and environmental samples. Biosecur Bioterror 2014; 12(1):49-62.

42. Gessler F, Pagel-Wider S, Avondet MA, Böhnel H. Evaluation of lateral flow assays for the detection of botulinum neurotoxin type A and their application in laboratory diagnosis of botulism. Diagn Microbiol Infect Dis 2007;57(3):243-249.

43. Iura K, Tsuge K, Seto Y, Sato A. Detection of proteinous toxins using the BioThreat Alert System. Japanese J Forensic Toxicol 2004;22:13-16.

44. Bertram-Sosa L, Jaso C, Valadez A, et al. Human plaguefour states, 2006. MMWR Morb Mortal Wkly Rep 2006; 55(34):940-943
45. Schotthoefer AM, Eisen RJ, Kugeler KJ, et al. Changing socioeconomic indicators of human plague, New Mexico, USA. Emerg Infect Dis 2012;18(7):1151-1154.

46. Morse SA, Kellogg RB, Perry S, Meyer RF, Nicholson D, Miller JM. Detecting biothreat agents: the Laboratory Response Network. ASM News 2003;69:433-437.

Manuscript received August 6, 2019;

revision returned October 14, 2019;

accepted for publication October 15, 2019.

Address correspondence to: Segaran P. Pillai, PhD

Director, Office of Laboratory Science and Safety FDA Office of the Commissioner Silver Spring, MD

Email: Segaran.Pillai@FDA.HHS.GOV 